\title{
Association between synovial fluid levels of inorganic pyrophosphate and short term radiographic outcome of knee osteoarthritis
}

\author{
Michael Doherty, Carolyn Belcher, Marian Regan, Adrian Jones, Joanna Ledingham
}

\begin{abstract}
Objective-To test the hypothesis that high concentrations of extracellular inorganic pyrophosphate (PPi), which associate with increased cell synthesis and turnover in cartilage, may act as a marker for structural outcome in knee osteoarthritis (OA).

Method-One hundred and thirty five consecutive patients referred to hospital with knee OA (59 men, 76 women; mean age 71 years, range 41-88) were followed prospectively for a median of 2.5 years (interquartile range $1 \cdot 75-3 \cdot 0)$. Synovial fluid (SF) aspirated at presentation (202 OA knees: 68 bilateral, 66 unilateral) was assessed for PPi content by radiometric assay. Knee radiographs at presentation and at final review were assessed for change in global (Kellgren) and individual features (narrowing, osteophyte, sclerosis, cyst, attrition) of $O A$.
\end{abstract}

Results-The median SF PPi level was 10.5 $\mu \mathrm{mol}$ (range 0.07-72.4). At baseline, high PPi was significantly associated with presence of calcium pyrophosphate crystals, chondrocalcinosis, and bone attrition. Radiographic change was observed in 164 knees. High PPi levels were negatively associated with change in Kellgren and Lawrence grade, further narrowing, and increase in osteophyte, but positively associated with development of attrition. In the 68 patients from whom bilateral data were obtained, there was correlation between right and left knees for PPi levels, all baseline radiographic scores, and changes in radiographic features. Multiple logistic regression analysis for $P P i$ as a continuous variable (age, gender, and patient number included in model) showed a negative correlation with change in global Kellgren and Lawrence grade (odds ratio (OR) $0.97,95 \%$ confidence interval (CI) 0.95 to 0.99 ) and a positive correlation with attrition (OR 1.04, 95\% CI 1.02 to 1.07).

Rheumatology Unit, City Hospital, Nottingham NG5 1PB, United Kingdom Correspondence to: Prof M Doherty, Rheumatology Unit, City Hospital, Nottingham NG5 1PB, United Kingdom

Accepted for publication 11 March 1996 outcome in terms of progressive change ellgren grade. Such elevated PPi level however, may inhibit new bone formation and remodelling in knee $O A$.

(Ann Rheum Dis 1996; 55: 432-436)
Inorganic pyrophosphate ( $\mathrm{PPi}$ ) is a by product of many essential biosynthetic reactions in mammalian cells. ${ }^{1}$ There is no recognised transmembrane transport mechanism for $\mathrm{PPi}$, and two mechanisms are postulated to contribute to extracellular PPi levels: first, leakage of intracellular $\mathrm{PPi}$ or coextrusion with other cellular products; ${ }^{1}$ second, and more importantly, salvage degradation of extracellular ATP by ectoenzymes, including nucleotide triphosphate pyrophosphohydrolase. ${ }^{1-8}$ Increased cellular activity and division may lead to elevated extracellular concentrations of PPi by either mechanism. ${ }^{1}$

In articular tissues, the chondrocyte and its associated vesicles are considered the principal source of extracellular PPi. ${ }^{1-4}$ Concentrations of PPi in knee synovial fluid (SF) are increased in osteoarthritis (OA) and in OA associated with calcium pyrophosphate dihydrate (CPPD) crystal deposition ('chronic pyrophosphate arthropathy'). ${ }^{5}$ Both these conditions are characterised by increased metabolic activity in cartilage and by new bone formation. ${ }^{9}$ In contrast, subnormal levels of SF PPi occur in knees affected by rheumatoid arthritis, ${ }^{7}$ a condition associated with marked attrition of cartilage and bone. Such observations suggest the possibility that increased concentrations of PPi resulting from increased biosynthetic activity may act as a marker for a potentially 'hypertrophic' (as opposed to 'atrophic') response to articular insult. ${ }^{79}$ If this were true, SF PPi levels might be expected to correlate with structural change and radiographic outcome in OA. This study was undertaken to test this hypothesis.

\section{Patients and methods}

The study was approved by the local research ethics committee. Consecutive patients referred with symptomatic knee $\mathrm{OA}$ to one general rheumatology clinic were recruited over a two year period. Details of these patients, part of a larger cohort, have been described previously. ${ }^{10} 11$ Alternative arthropathies were excluded by clinical examination, blood tests (serology, acute phase response), microscopy of $S F$, and radiographic screening. ${ }^{11}$ Knee OA was defined radiographically, from standing fully extended anteroposterior and $30^{\circ}$ flexion lateral views, as presence of joint space narrowing with osteophyte, sclerosis, or both, in at least one knee compartment (patellofemoral, medial tibiofemoral, lateral tibiofemoral). 
ASSAY OF SYNOVIAL FLUID INORGANIC PYROPHOSPHATE

All OA knees were aspirated at presentation, irrespective of whether an effusion was detected clinically. A sample of each SF was examined fresh, by compensated polarised light microscopy, for presence of CPPD crystals (identified by characteristic morphology and birefringence).

For the PPi assay, SF was taken into sterile plastic containers on ice, spun at $2500 \mathrm{~g}$ for 15 minutes at $4^{\circ} \mathrm{C}$, and the resulting supernatant stored frozen at $-20^{\circ} \mathrm{C}$. SF PPi levels were estimated using a modification ${ }^{7}$ of a specific, sensitive radiometric assay. ${ }^{12}$ In brief, the SF sample PPi was allowed to react with tritiated uridine diphosphogluconate, and the product, glucose-1-phosphate, was converted to a labelled, stable, recoverable product (tritiated 6-phosphogluconate) estimated by radioactivity count (each sample assayed in triplicate). To measure any hydrolysis of PPi to orthophosphate during incubation and extraction, a recovery procedure was undertaken (performed in duplicate) involving addition of tracer $\left[{ }^{32} \mathrm{P}\right] \mathrm{PPi}$ to the initial sample, removal of orthophosphate by complexing with ammonium molybdate and recovery into isobutanol/light petroleum, and counting of the remaining $\left[{ }^{32} \mathrm{P}\right] \mathrm{PPi}$ in the aqueous phase.

\section{RADIOGRAPHIC ASSESSMENTS}

Standardised plain radiographs of knees were obtained at the patient's entry to the study and then at least annually. Study films were a standing anteroposterior radiograph of the tibiofemoral joints $(55 \mathrm{kV}, 8 \mathrm{~mA}$ s, full scale deflection (FSD) $100 \mathrm{~cm}$ ) plus lateral views in $30^{\circ}$ flexion of the patellofemoral joint $(55 \mathrm{kV}$, $8 \mathrm{~mA} \mathrm{~s}$, FSD $100 \mathrm{~cm}$ ). Initial films were examined and scored by two observers as described previously. ${ }^{10}{ }^{11}$ Each knee compartment was given a global radiographic score for OA using a system based on the Kellgren and Lawrence scale. ${ }^{13}$ In addition, individual features of OA (narrowing, osteophyte, sclerosis, cysts) were graded on a scale of 0-3 using a modification of the method described by Thomas et al. ${ }^{14}$ Chondrocalcinosis (calcification in hyaline or fibrocartilage) and attrition

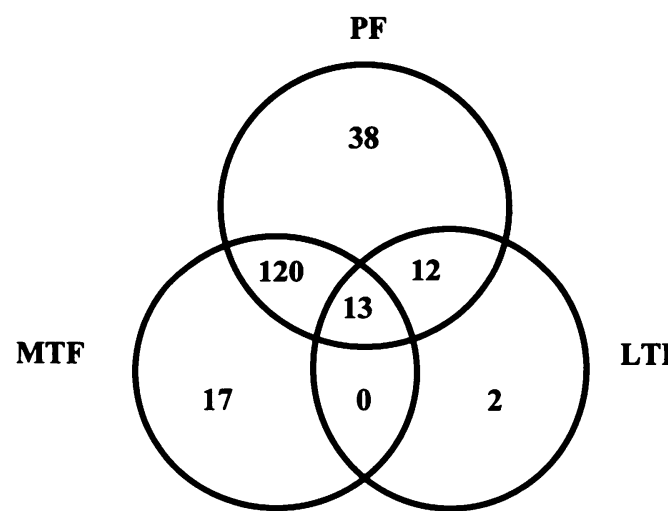

Compartmental distribution of OA change in the 202 study knees from which synovial fluid for PPi estimation was obtained. $P F=$ Patellofemoral; $M T F=$ medial tibiofemoral; LTF = lateral tibiofemoral. (loss/collapse of subchondral bone stock resulting in apparent reduced bone volume) were each recorded as present or absent. The last available knee radiographs were assessed in a manner identical to that applied to the study entry film (blinded to the original assessment). Individual radiographic features (narrowing, osteophyte, cysts, sclerosis, attrition) were assessed for change (increase, same, decrease) at each knee compartment, by comparison of scores from initial and final radiographs. Reproducibility (for the same assessors) for the scoring of individual radiographic features and Kellgren grade, and for the scoring of change in individual radiographic features had previously been demonstrated to be good. ${ }^{1011}$

\section{STATISTICAL ANALYSIS}

Statistical analysis was by the Mann Whitney $U$ test, logistic regression (Egret, Serc, Seattle), Spearman rank correlation, and the kappa ( $\kappa$ ) statistic as appropriate. The $\kappa$ statistic was used as a measure of association-that is, as a test of symmetry over and above that expected by chance. Correlation between results from right and left knees in those with bilateral data was undertaken on Statpak using the correlation and regression programme; because correlations were found, a dummy patient variable was included in the regression model to take this into account.

\section{Results}

One hundred and thirty five consecutive patients (76 women, 59 men; mean age 71 years, range 41-88) were recruited. Knee OA was bilateral in $117(87 \%)$. An amount of SF sufficient for estimation of $\mathrm{PPi}$ levels was obtained from 202 (81\%) OA knees (134 patients) - specifically, from both knees of 68 patients and from one knee of 66 patients.

\section{RADIOGRAPHIC FINDINGS}

The figure shows the compartmental distribution of OA change (any of the specified OA features) for the 202 study knees at entry, and table 1 lists the mean summated (medial and lateral tibiofemoral, patellofemoral compartments) radiographic scores at entry for the various measures. Chondrocalcinosis was present in $70(34 \%)$ knees (43 patients), and CPPD crystals were identified in 68 (33\%) knees (40 patients).

In the 68 patients for whom bilateral knee SF data were obtained, there was close correlation between radiographic findings at baseline in

Table 1 Summated radiographic scores (patellofemoral and lateral and medial tibiofemoral compartments) for various features

\begin{tabular}{llll}
\hline Radiographic feature & Mean & Median & Range \\
\hline Kellgren \& Lawrence grade & 3.67 & 4 & $1-8$ \\
Narrowing & 3.67 & 4 & $1-8$ \\
Osteophyte & $4 \cdot 08$ & 4 & $0-9$ \\
Cysts & $1 \cdot 12$ & 1 & $0-6$ \\
Sclerosis & $1 \cdot 70$ & 2 & $0-6$ \\
Attrition & $0 \cdot 75$ & 0 & $0-4$ \\
\hline
\end{tabular}


Table 2 Correlation between right and left knees for radiographic features at baseline and for change with time in 68 patients for whom there were bilateral knee data

\begin{tabular}{|c|c|c|}
\hline \multirow[t]{2}{*}{ Radiographic feature correlation } & $\underline{\text { Baseline }}$ & \multirow{2}{*}{$\frac{\text { Change with time }}{\mathrm{r}^{2}}$} \\
\hline & $\overline{r^{2}}$ & \\
\hline Summated (total knee) Kellgren grade & $0 \cdot 68$ & 0.43 \\
\hline Summated patellofemoral score & 0.73 & $0 \cdot 38$ \\
\hline Summated medial tibiofemoral score & 0.69 & $0 \cdot 38$ \\
\hline Summated lateral tibiofemoral score & 0.62 & $0 \cdot 83$ \\
\hline $\begin{array}{l}\text { Summated (three compartment) score } \\
\text { for: }\end{array}$ & & \\
\hline Narrowing & $0 \cdot 68$ & 0.50 \\
\hline Osteophyte & 0.73 & $0 \cdot 28$ \\
\hline Cyst & 0.65 & $0 \cdot 30$ \\
\hline Sclerosis & 0.44 & 0.57 \\
\hline Attrition & $0 \cdot 70$ & $0 \cdot 47$ \\
\hline
\end{tabular}

right and left knees with respect to compartmental distribution, summated Kellgren scores and scores for individual features (table 2).

The median duration of follow up was 2.5 years (interquartile range $1 \cdot 75-3 \cdot 0$ ). Over this period, 164 knees showed change in at least one radiographic feature, and 38 knees showed no change. Table 3 shows the frequency of change in each radiographic feature during the study. In the 68 patients with bilateral data, there was close agreement between right and left knees for change in each radiographic feature (table 2); for example, for increase in Kellgren grade the correlation was 0.43 , with a $\kappa$ of 0.71 , and odds ratio of $6.75(95 \% \mathrm{CI}$ 1.97 to $24 \cdot 24)$.

SPECIFICITY, SENSITIVITY AND VARIABILITY OF PPi ASSAY

The limit of detection of the PPi assay was 100 pmol of PPi. Specificity was demonstrated by significant loss of radioactivity on addition of yeast pyrophosphatase to the final incubation. Recovery of PPi added to SF (in triplicate measurements) ranged from $92 \%$ to $105 \%$ when concentrations of added PPi ranged from 0.5 to $8 \mu \mathrm{mol}$. The coefficient of variation for the assays was $9 \%$ (same day estimations on 10 aliquots of the same SF), and the mean coefficient of variation $(n=10)$ between triplicate measurements was $8 \%$.

CROSS SECTIONAL DATA AT PRESENTATION A wide range of values of SF PPi was found (median $10.5 \mu \mathrm{mol}$, range $0.07-72.40$ ). In the 68 patients for whom there were bilateral SF data, there was correlation between levels in right and left knees $\left(r^{2}=0 \cdot 84\right)$. Levels were significantly greater in women than in men (median $13.0 \mu \mathrm{mol}$ (95\% CI 9.3 to 14.8 ) compared with $7 \cdot 2 \mu \mathrm{mol}(95 \%$ CI 6.6 to $9 \cdot 8$ ), respectively) $(\mathrm{p}<0.01)$ and increased significantly with increasing age of the subject (median $9 \cdot 1 \mu \mathrm{mol}(95 \%$ CI $7 \cdot 2$ to $12 \cdot 8$ ) for age $<80$ years, compared with $14.3 \mu \mathrm{mol}$ (95\% CI

Table 3 Frequency of change in radiographic features of knee osteoarthritis

\begin{tabular}{lll}
\hline Change in radiographic feature & No of knees & $(\%)$ \\
\hline Further joint space narrowing & 120 & 59 \\
Increase in osteophyte & 65 & 32 \\
Increase in cyst & 44 & 21 \\
Increase in sclerosis & 36 & 18 \\
Development of attrition & 44 & 22 \\
Increase in Kellgren grade & 86 & 46 \\
\hline
\end{tabular}

11.9 to 16.3$)$ for age $>80$ years $)(p<0.01)$ High levels of PPi were associated with presence of CPPD crystals (median levels for presence and absence $18.5 \mu \mathrm{mol}(95 \%$ CI 15.8 to $25 \cdot 0)$ compared with $7 \cdot 4 \mu \mathrm{mol}(95 \% \mathrm{CI} 6 \cdot 3$ to $9 \cdot 3)$, respectively), chondrocalcinosis (median $16.0 \mu \mathrm{mol}(95 \%$ CI 13.4 to $24 \cdot 7$ ) compared with $8.0 \mu \mathrm{mol}$ (95\% CI 6.6 to $10 \cdot 7)$ ), and bone attrition (median $14.8 \mu \mathrm{mol}$ (95\% CI 13.0 to 18.6 ) compared with $7 \cdot 8$ $\mu \mathrm{mol}(95 \%$ CI 6.6 to 9.6$))(\mathrm{p}<0.001)$. No association was found with presence of joint space loss, osteophyte, sclerosis, or cyst formation.

PROSPECTIVE DATA

There was no difference in PPi levels between the 38 knees showing no radiographic change (median $11.3 \mu \mathrm{mol}, 95 \%$ CI 7.2 to 15.6 ) and the 164 showing some change (median 10.4 $\mu \mathrm{mol}, 95 \%$ CI $7 \cdot 7$ to $13 \cdot 7$ ). However, associations were noted for changes in individual radiographic features. Lower levels of SF PPi associated with further decrease in joint space and change in osteophyte: median levels 8.8 $\mu \mathrm{mol}(95 \%$ CI $7 \cdot 2$ to 12.4$)$ compared with $12.6 \mu \mathrm{mol}(95 \%$ CI 7.8 to 14.7$)(\mathrm{p}<0.01)$, and $7 \cdot 2 \mu \mathrm{mol}(95 \%$ CI 5.9 to $9 \cdot 1)$ compared with $12.7 \mu \mathrm{mol}(95 \%$ CI $9 \cdot 1$ to 15.0$)$ $(\mathrm{p}<0.001)$ in knees with and without change, respectively.

Table 4 shows results for change in Kellgren grade and change in individual radiographic features when PPi was examined as a continuous variable, and when PPi levels were grouped as quartiles, for which the ranges of values were: $0.07-5.5 \mu \mathrm{mol}$ (1st quartile); $5 \cdot 6-10 \cdot 5 \mu \mathrm{mol}$ (2nd quartile); $10 \cdot 6-16 \cdot 9 \mu \mathrm{mol}$ (3rd quartile); $17 \cdot 0-72 \cdot 4 \mu \mathrm{mol}$ (4th quartile).

High levels of PPi associated negatively with increase in Kellgren scores and increase in osteophyte, but positively with development of attrition. No linear association between PPi levels and change in either narrowing or cyst was discerned; for these two features both very low (1st quartile) and very high (4th quartile) PPi levels showed greatest tendency to change. Low levels of PPi associated with change in sclerosis. However, when logistic regression was used to examine PPi as a continuous variable, with age, gender, and 'dummy' patient variable included in the model, two of these associations remained: the negative association with change in Kellgren score (odds ratio (OR) 0.97, 95\% CI 0.95 to 0.99 ), and the positive association with development of attrition (OR 1.04, 95\% CI 1.02 to 1.07$)$. A negative association between age and change in osteophyte (OR 0.97, $95 \%$ CI 0.94 to 0.99 ), but a positive association between age and attrition (OR $1.05,95 \% \mathrm{CI}$ 1.04 to 1.09 ) were also observed. No associations were found between PPi and change in osteophyte, cyst, or sclerosis.

\section{Discussion}

There is considerable interest in putative 'markers' of OA, with most attention focusing on components of cartilage, synovium, or bone 
Table 4 PPi levels and change in radiographic features

\begin{tabular}{|c|c|c|c|c|c|}
\hline & $\begin{array}{l}\text { \% with } \\
\text { change }\end{array}$ & $\begin{array}{l}\chi^{2} \text { test for } \\
\text { trend }\end{array}$ & $p$ & $\begin{array}{l}\text { Odds } \\
\text { ratio }\end{array}$ & $95 \% C I$ \\
\hline \multicolumn{6}{|l|}{ Kellgren \& Lawrence grade } \\
\hline $\begin{array}{l}\text { PPi (continuous variable) } \\
\text { PPi quartiles }\end{array}$ & & & & 0.97 & 0.95 to 0.99 \\
\hline $\begin{array}{l}\text { PPi quartiles } \\
\text { 1 st }\end{array}$ & 29 & $4 \cdot 31$ & $0 \cdot 038$ & $1 \cdot 0$ & \\
\hline 2nd & 29 & & & 1.09 & 0.46 to 2.59 \\
\hline $3 \mathrm{rd}$ & 22 & & & 0.54 & 0.23 to 1.28 \\
\hline 4th & 20 & & & 0.52 & 0.21 to 1.24 \\
\hline \multicolumn{6}{|l|}{ Osteophyte } \\
\hline $\mathrm{PPi}$ (continuous variable) & & & & 0.99 & 0.96 to 1.01 \\
\hline PPi quartiles & & $9 \cdot 19$ & $0 \cdot 002$ & & \\
\hline $1 \mathrm{st}$ & 35 & & & $1 \cdot 0$ & \\
\hline 2nd & 31 & & & 0.84 & 0.35 to $2 \cdot 01$ \\
\hline $3 \mathrm{rd}$ & 15 & & & $0 \cdot 27$ & $0 \cdot 10$ to $0 \cdot 70$ \\
\hline 4th & 18 & & & 0.37 & 0.14 to 0.94 \\
\hline \multicolumn{6}{|l|}{ Attrition } \\
\hline $\mathrm{PPi}$ (continuous variable) & & & & 1.04 & 1.02 to 1.07 \\
\hline PPi quartiles & & $8 \cdot 37$ & 0.003 & & \\
\hline $1 \mathrm{st}$ & 14 & & & $1 \cdot 0$ & \\
\hline 2nd & 18 & & & 1.47 & 0.41 to 5.30 \\
\hline 3rd & 27 & & & $2 \cdot 10$ & 0.65 to 6.98 \\
\hline 4th & 41 & & & $4 \cdot 13$ & 1.34 to 13.2 \\
\hline \multicolumn{6}{|l|}{ Narrowing } \\
\hline $\mathrm{PPi}$ (continuous variable) & & & & 1.02 & 0.99 to 1.04 \\
\hline PPi quartiles & & $1 \cdot 72$ & $0 \cdot 19$ & & \\
\hline $1 \mathrm{st}$ & 25 & & & $1 \cdot 0$ & \\
\hline 2nd & 20 & & & 0.67 & 0.28 to 1.60 \\
\hline 3 rd & 25 & & & 0.83 & 0.35 to 1.96 \\
\hline 4 th & 30 & & & $1 \cdot 71$ & 0.68 to 4.32 \\
\hline \multicolumn{6}{|l|}{ Cysts } \\
\hline $\mathrm{PPi}$ (continuous variable) & & & & $1 \cdot 02$ & \\
\hline PPi quartiles & & 0.67 & $0 \cdot 41$ & & \\
\hline $1 \mathrm{st}$ & 32 & & & $1 \cdot 0$ & \\
\hline 2nd & 11 & & & $0 \cdot 30$ & 0.08 to 1.01 \\
\hline 3rd & 18 & & & 0.45 & 0.15 to 1.30 \\
\hline 4 th & 39 & & & $1 \cdot 32$ & 0.14 to 0.94 \\
\hline \multicolumn{6}{|l|}{ Sclerosis } \\
\hline PPi (continuous variable) & & & & 0.99 & 0.96 to 1.01 \\
\hline PPi quartiles & & $4 \cdot 97$ & 0.03 & & \\
\hline lst & 38 & & & $1 \cdot 0$ & \\
\hline 2nd & 23 & & & 0.51 & 0.20 to 1.30 \\
\hline 3rd & 18 & & & $0 \cdot 31$ & 0.12 to 0.83 \\
\hline 4 th & 21 & & & 0.44 & 0.17 to 1.12 \\
\hline
\end{tabular}

$\mathrm{CI}=$ Confidence interval. loss of interosseous distance in tibiofemoral compartments, ${ }^{1+18}$ and lateral flexion views for the patellofemoral compartment. Although we recently reported that the 'skyline' view has certain advantages over the lateral flexion view, ${ }^{19}$ we were using lateral views alone at the start of this study. The same technique and film were used for all radiographs, and a large number of knees were studied. The global scoring system of Kellgren and Lawrence ${ }^{13}$ is commonly used, but assumes that different features of OA progress together. Separate grading of individual features ${ }^{14}$ is less commonly used, but may be more sensitive to change, ${ }^{11}$ and assumes no particular hierarchy with respect to progression. We therefore used both scoring systems in this study, having shown good intra- and interobserver reproducibility for both systems in this same study population. $^{10} 11$ We found a negative association between PPi levels and change in Kellgren grade, but different (negative and positive) associations for individual features. This finding alone supports the need to consider individual features in outcome 'marker' studies. It may also explain in part the lack of difference between knees showing any change and knees with no change, the separate negative and positive associations tending to cancel out and reduce the group difference.

An interesting finding in the patients who contributed bilateral knee data was the positive correlation, both for radiographic scores and SF PPi levels, between right and left knees at baseline. Furthermore, the tendency to change in radiographic features in right and left knees also correlated. This lack of independence presents certain problems for analysis, which are only rarely considered in such studies. Clinically, there is interest in both knees with respect to outcome; exclusion of one knee (for example the least 'worst') for the purposes of statistical analysis therefore seems inappropriate. Having found evidence for dependency between right and left data in the same patient, we elected to correct for this by including a dummy patient-dependent variable in the logistic regression model. However, other methods of tackling this problem have been suggested recently. ${ }^{20}$

There are a number of important caveats to this study. First, we estimated SF PPi at a single time point and did not undertake serial estimations to assess fluctuations or trends over time. Second, because all patients had been referred to hospital, they may have represented a population with a more severe form of OA. Further study is required to establish to what extent these findings may be generalised to early, less severe OA. Third, these data relate to radiographic change alone. Multiple factors, including psychosocial ones, influence the severity of pain and disability in knee OA, ${ }^{21} 22$ and control for such multiple factors was impractical for a study this size. The radiometric assay that we used for estimation of PPi is sensitive and specific, and can be applied to small SF volumes. However, the assay is complex and expensive in terms of materials and technician time. As with any SF assay, the OA. Plain radiographs have several inheren problems, for example standardisation of positioning, magnification, and film quality, insensitivity for change, and problems with observer error. In this study we used standardised standing films to achieve better visualisation of 
concentration measured depends on a number of variables including rate of synthesis, rate of dissolution, and SF clearance. We have shown previously that SF PPi levels in knee OA are not influenced by the degree of clinically assessed inflammation, though this can be an issue in OA knees with CPPD crystal deposition. ${ }^{\top}$ In the present study, greater SF levels of PPi occurred in women and in older subjects, perhaps reflecting more widespread compartmental involvement and tendency to CPPD deposition/chondrocalcinosis in these individuals. PPi levels may relate to deposition of both CPPD and calcium phosphate crystals, both of which commonly deposit in OA knees and have been suggested to associate with more severe, more widespread multicompartmental $\mathrm{OA}^{1023}{ }^{24}$ and tendency to radiographic progression. ${ }^{11}$ Whether such crystal deposition is cause or consequence of OA change, however, remains unclear. Crystal formation, growth, dissolution, and trafficking within joints are complex phenomena, ${ }^{9}$ and identification and quantification of SF crystals are extremely problematic. Clear associations of calcium crystals with $\mathrm{OA}$ and SF PPi are therefore difficult to define.

PPi metabolism is incompletely understood, and these data are open to several interpretations. If cellular activity is an important influence tending, directly or indirectly, to increase extracellular levels of $\mathrm{PPi}^{1-9}$ then increased SF levels might be expected to associate with little progression of structural change ('compensated' OA). Conversely, low levels of SF PPi, reflecting poor cellular (predominantly chondrocyte) activity would be expected to associate with progression of structural change ('decompensated' OA). Extracellular concentrations of PPi can modulate cartilage and bone mineralisation, with moderate increase stimulating, but gross increase inhibiting, calcium phosphate crystal deposition. ${ }^{125-28}$ Increased PPi levels that accompany exuberant cellular activity may therefore inhibit apatite deposition and bone remodelling, and thus associate with little change in osteophyte and a tendency to bone attrition. Of special interest was the finding that both very high and very low levels of PPi might associate with further joint space narrowing. This could be explained if very high levels result mainly from release of intracellular $\mathrm{PPi}$ as a result of cell death or damage.

In summary, this prospective study of hospital referred patients with knee OA supports an association between SF concentrations of PPi at presentation and tendency to undergo further radiographic change over the next two to three years. Further investigation of SF PPi as a potential marker of the OA process seems warranted.

JL is a Roussel (UK) Osteoarthritis Research Fellow. We are grateful to Trent Regional Health Authority and the Arthritis and Rheumatism Council for financial support.

1 Rachow J W, Ryan L M. Inorganic pyrophosphate metabolism in arthritis. Rheum Dis Clin North Am 1988 14: $289-302$.
2 Muniz O, Pelletier J-P, Martel-Pelletier J, . Morales S, Howell D S. NTP pyrophosphohvdrolase in human chondrocalcinotic and osteoarthritic cartilage. Attlyiti Rheum 1984; 27: 186-92.

3 Howell D S, Martel-Pelletier J, Pelletier J-P, Morales S. Muniz O. NTP prophosphohidrolase in human chondrocalcinotic and osteoarthritic cartilage. II. Further studies on histologic and subcellular distribution. Arthitis Studies on histologic and

4 Rvan L. M, Wortmann R L, Karas B, McCarty D J. Cartilage nucleoside triphosphate prrophosphohydrolase. II. Rol in extracellular pyrophosphate generation and nucleotide metabolism. Arthitis Rheum 1985; 28: $113-7$.

5 Rachow J W', Ryan L. M. Adenosine triphosphate pyrophosphohydrolase and neutral inorganic pyrophosphatase in pathologic joint fluids. Arthritis Rheum 1985; 28: 1283-8

6 Rvan L . M, Rachow J W', McCarty D J. Sinovial tluid ATP: a potential substrate for the production of inorganic a potential substrate for the production of

7 Pattrick M. Hamilton E, Hornby J, Doherty M. Synovial fluid pyrophosphate and nucleoside triphosphate pyrophosphatase: comparison between normal and diseased and between inflamed and non-inflamed joints. Am Rhcum Dis 1991; 50: 214-8.

Terkeltaub R, Rosenbach M, Fong F, Goding J. Causal link between nucleotide pyrophosphohydrolase oreractivity and increased intracellular inorganic pyrophosphat generation demonstrated by transfection of cultured fibroblasts and osteoblasts with plasma cell membrane glycoprotein-1. Arthritis Rhutum 1994; 37: 934-41.

9 Doherty M. Dieppe P A. Clinical aspects of calcium pyrophosphate dihydrate cristal deposition. Rhitum Di Clin N Ain 1988; 1 i: 395-41.

10 Ledingham I M. Regan M, Jones A, Doherty M. Radiographic patterns and associations of knee osteoarthritis in a hospital referred population. Ann Rhillm Dis 1993; 52 263.

11 Ledingham J M, Regan M, Jones A, Doherty .11. Factor affecting radiographic progression of knee osteoarthritis Ann Rhelum Dis 1995; 54: 53-8.

12 McGuire M B, Colman C H, Baghat $\mathrm{X}$, Russel R G G Radiometric measurement of pyrophosphate in cell cultures. Biochem Soc Trans 1980; 8: 529-30.

13 Kellgren J H, Lawrence J S. Radiological assessment of osteoarthritis. Am Rheum Dis 1957;16: 194-502.

1+ Thomas R H, Resnick D, Alazraki \& P, Davies D, Greenfield R. Compartmental evaluation of osteoarthritis of the knee. A comparative study of available diagnostic modalities. Diagn Radiol 1975; 16: 585-94.

15 Poole R. Immunochemical markers of joint inflammation damage and repair: where are we now? Ann Rhicum $D i$ 1994; 53: 3-5.

16 Ryan L M, Cheung H S, McCarty D J. Release of prrophosphate by normal mammalian hvaline and fibrocartilage. Arthritis Rheum 1981; 24: 1522-7.

17 Hamilton E, Pattrick M, Doherty M. Inorganic pyrophosphate, nucleoside triphosphate pyrophosphatase, and cartilage fragments in normal human synovial fluid. $\mathrm{Br} / \mathrm{f}$ Rheumatol 1991; 30: 260-4.

18 Leach R E, Gregg T, Siber F J. Weight bearing radiography in osteoarthritis of the knee. Radiology 1970; 97: 265-8.

19 Jones A, Ledingham J, McAlindon T, et al. Radiographic assessment of patellofemoral osteoarthritis. Amm Rhim Dis 1993; 52: 655-8.

20 Zhang Y Q, Glynn R J, Felson D T. Musculoskeletal disease research: should we analyse the joint or the person Arthritis Rheum 1995; 38(suppl): S17T.

21 Summers M N, Haley W E, Reveille J D, Alarcon G S. Radiographic assessments and psychologic variables as predictors of pain and functional impairment in osteoarthritis of the knee or hip. Arthritis Rheum 1988; 31 . $204-9$.

22 Davis M A, Ettinger W H, Neuhaus J M, Barclav J D, Segal M R. Correlates of knee pain among US adults with and without radiographic knee osteoarthritis. 7 Rhetmatol 1992; 19: 1943-9.

23 Rachow J W, Ryan I, M, McCarty D J, Halverson P Synovial fluid inorganic pyrophosphate concentration and nucleotide pyrophosphohydrolase activity in basic calcium phosphate deposition arthropathy and Milwaukee shoulder syndrome. Arthritis Rheum 1988; 31 : 408-13.

24 Pattrick M, Hamilton E, Wilson R, Austin S, Doherty M. Association of radiographic changes of osteoarthritis, symptoms and synovial fluid particles in 300 knees. Aut Rheum Dis 1993; 52: $97-103$.

25 Fleisch H, Strauman F, Schenk R, Bisaz S, Allgower M Effect of condensed phosphates on calcification of chick embryo femurs in tissue culture. Am $\mathcal{f}$ Physiol 1966;211: $821-5$.

26 Seigel S A, Hummel C F, Cart R P. The role of nucleoside triphosphate pyrophosphohydrolase in in vitro nucleoside triphosphate-dependent matrix vesicle calcification. $7 \mathrm{Biol}$ Chem 1983; 258: 8601-7.

27 Hsu H H T, Anderson H C. The deposition of calcium pyrophosphate and phosphate by matrix vesicle isolated from fetal bovine epiphyseal cartilage. Calcif Tissue In 1984; 36: 615-21

28 Einhorn T A, Gorden S L, Siegel S A, Hummel C F, Avitable M J, Carty R P. Matrix vesicle enzymes in human osteoarthritis. F Orthop Res 1985; 3: 160-9. 